\title{
The role of early adversity and cognitive vulnerability in postnatal stress and depression
}

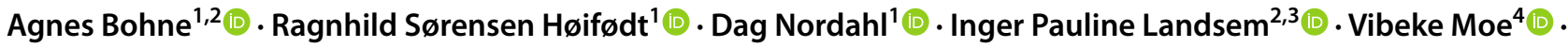 \\ Catharina Elisabeth Arfwedson Wang ${ }^{1}$ (i) . Gerit Pfuhl ${ }^{1}$ (i)
}

Accepted: 20 December 2021

(c) The Author(s) 2022

\begin{abstract}
The purpose of the present study was to examine vulnerability factors in expecting parents that might lead to mental illness in the perinatal period. Specifically, we studied how parental early adversity, attentional bias to infant faces, repetitive negative thinking, and demographic factors, were associated with pre- and postnatal depressive symptoms and parenting stress. Participants were expecting parents taking part in the Northern Babies Longitudinal Study, where assessments were made both pre- and postnatally. Assessments included both questionnaires and cognitive tasks. About half of the participants received the Newborn Behavior Observation (NBO)-intervention after birth, between pre- and postnatal assessments. Results show that repetitive negative thinking was a significant predictor of both depressive symptoms and parenting stress, while education, social support, and parity came out as protective factors, especially in mothers. Parental early adversity had an indirect effect on postnatal depressive symptoms and parenting stress, mediated by prenatal and postnatal depressive symptoms, respectively. The NBO intervention did not affect the results, signifying the importance of early childhood adverse events and negative thinking on parents' postnatal adjustment and mood, even when an intervention is provided. In conclusion, repetitive negative thinking is a significant vulnerability factor independent of the presence of depressive symptoms, and health professionals must be aware of parents' thinking style both during pregnancy and after birth.
\end{abstract}

Keywords Perinatal depression $\cdot$ Parental stress $\cdot$ Repetitive negative thinking $\cdot$ Adverse childhood experience $\cdot$ Attentional bias $\cdot$ Cognitive thinking style

\section{Introduction}

Becoming a parent is among the greatest transitions in life, and a vulnerable period. The perinatal period is associated with increased risk of depression and symptoms of mental illness in parents (Bauer et al., 2014). Mental illness and psychosocial difficulties in parents are not only distressing for the parents themselves, but also a risk factor for infant

Agnes Bohne

agnes.bohne@uit.no

1 Department of Psychology, Faculty of Health Sciences, UiT The Arctic University of Norway, N-9037 Troms $\varnothing$, Norway

2 Division of Child and Adolescent Health, University Hospital of Northern Norway, Troms $\varnothing$, Norway

3 Department of Health and Care Sciences, Faculty of Health Sciences, UiT The Arctic University of Norway, Troms $\varnothing$, Norway

4 Department of Psychology, University of Oslo, Oslo, Norway development, as they may affect the quality of parenting (Goodman et al., 2011). To develop effective interventions for struggling families, it is essential to identify the factors contributing to parental stress and mental illness in the perinatal period. The purpose of the present study was to examine the role of parental early adversity and cognitive vulnerability factors in this period, as previous research regarding those factors is sparse.

\section{Influences of Early Adversity}

The Adverse Childhood Experiences-study (ACE-study) demonstrated how adverse experiences like abuse, neglect, and dysfunctional home-situations during childhood increased the risk of a whole range of mental and somatic illnesses in adult life (Felitti et al., 1998). Among them, the relationship between experiencing childhood maltreatment and later symptoms and diagnosis of depression is well established (Alloy et al., 2006). In the perinatal period, 
maternal ACE is found to be a risk factor for both perinatal depression (Racine et al., 2020) and parenting stress (Moe et al., 2018). In fathers, ACE is related to prenatal anxiety and depression (Skjothaug et al., 2014). Maternal ACE can not only affect the mother's own health, but also their infant's health (Esteves et al., 2020). This highlights how risk can transfer across generations, creating a vicious cycle that can be hard to break.

\section{Influences of Negative Cognitions}

Cognitive processing biases are considered an important factor in the onset and maintenance of depression (reviewed in LeMoult \& Gotlib, 2019). Cognitive theories of depression posit that negative cognitions, like repetitive negative thinking or selective attention to negative stimuli, may represent psychological vulnerability (Joormann, 2010). Vulnerability can be defined as a stable underlying factor in the individual that makes them less resistant to stress (Ingram \& Luxton, 2005). In interaction with a stressor (e.g., an adverse experience or a transitional period like the perinatal period) depression may be triggered (Ingram \& Luxton, 2005). Furthermore, there are indications that attentional bias is related to perinatal depression and parenting. For example, expecting mothers who were more attentive to distressed infant faces during pregnancy reported more successful mother-infant bonding (Pearson et al., 2011). Expecting mothers with depressive symptoms were less attentive to emotional infant faces (Pearson et al., 2010, 2013). Thompson-Booth et al. (2014) found aberrant attention to emotional infant faces in parents with higher levels of parenting stress.

In addition, rumination and repetitive negative thinking prenatally is related to higher levels of postnatal depressive symptoms (Barnum et al., 2013; Schmidt et al., 2017). Rumination is traditionally referred to as a characteristic of depression, while repetitive negative thinking is considered transdiagnostic (Ehring et al., 2011). However, several authors refer to them interchangeably in the literature (e.g. DeJong et al., 2016). Repetitive negative thinking during pregnancy is also negatively associated with mother-infant bonding after birth (Müller et al., 2013; Schmidt et al., 2017). Interestingly, Müller et al. (2013) found no association between repetitive negative thinking during pregnancy and postnatal depressive symptoms. In their review, DeJong et al. (2016) concluded that the evidence of rumination predicting later postnatal depression is mixed, and rumination might not be predictive when controlling for concurrent and previous depressive episodes. To avoid possible confounding of measuring both depression and rumination, the present study applied a measure of repetitive negative thinking. However, rumination and repetitive negative thinking are highly related; accordingly, the literature on rumination is relevant too.

\section{Postnatal Depression and Parenting Stress}

Postnatal depression is prevalent in about $10-15 \%$ of mothers (Bauer et al., 2014; Ramchandani et al., 2005). Postnatal depression serves as a risk factor for insecure infant attachment, and could negatively affect mother-infant interactions (Tronick \& Reck, 2009) and infant development (Goodman et al., 2011). Most research in this area has focused on mothers, but postnatal depression is a significant problem for fathers as well, and is associated with adverse child outcomes (Ramchandani et al., 2005). Fathers' mental health in the perinatal period is undeniably important and accordingly the present study recruited both expecting mothers and their partners.

Parenting stress arises when parents perceive that the demands of parenting exceeds their resources (Abidin, 1992). In mothers, anxiety and prenatal and postnatal depression are associated with parenting stress after birth (Leigh \& Milgrom, 2008; Mazzeschi et al., 2015). In fathers, prenatal mental health predicted paternal stress 6 months after birth (Skjothaug et al., 2018). Parenting stress is associated with both the externalizing and internalizing of problems in the child (Barroso et al., 2018; Fredriksen et al., 2018), but also with relational problems between the parents (Mazzeschi et al., 2015). Thus, higher levels of parenting stress have adverse effects on the parents' mental health, the parent-child relationship and the child's development. Therefore, it is paramount to understand what leads to experiencing high levels of parenting stress.

\section{Protective Factors}

For most couples, pregnancy and childbirth are a positive experience where expecting parents experience social support, which in turn is protective against prenatal depressive symptoms (Leigh \& Milgrom, 2008; Racine et al., 2020), parenting stress (Chich-Hsiu et al., 2011; Östberg \& Hagekull, 2000; Racine et al., 2019) and postnatal depressive symptoms (Racine et al., 2020). Age is another possible protective factor. Bergström (2013) found that fathers of higher age had less risk of postnatal depressive symptoms than younger fathers, even when sociodemographic factors had been accounted for. The data are mixed for mothers, where some studies reported a protective effect while others reported no effect or an adverse effect of higher age on depression (Norhayati et al., 2015). Similarly, the association between postnatal depressive symptoms and education level varies between studies (Norhayati et al., 2015). 


\section{The Newborn Behavioral Observation (NBO) Intervention}

The struggles experienced by many families in the postnatal period call for health care services to empower new parents and reduce stress and symptoms of mental illness. The Newborn Behavioral Observation (NBO) is a consultation where the parents together with a health professional observe the infant's competencies, behavioral repertoire, and communication cues. The goal is to sensitize the parents to their infant's communication and provide tailored supervision, and in this way increase parental competence and confidence (Nugent, 2007). There are a few studies yielding encouraging results, where the NBO is found to increase maternal engagement (Sanders \& Buckner, 2006) and sensitivity (Nugent et al., 2017). However, in the Northern Babies Longitudinal Study (NorBaby; Høifødt et al., 2017), which the present study was part of, no effect of the NBO was found on maternal depressive symptoms or stress (Høifødt et al., 2020). In line with these findings, we did not expect the NBO to affect the results in 8 . However, the current study may shed light on how interventions like NBO can be changed in order to make them more effective.

\section{The Present Study}

The present study aimed to add more knowledge about which parental vulnerability factors predict postnatal depression and parenting stress. Previous research on cognitive vulnerability factors has focused on mothers. Thus, to our knowledge, our study was the first to investigate not only the role of mothers', but also fathers' early adversity and cognitive styles on postnatal depression and parenting stress. The present study hypothesized that adverse childhood experiences, attentional bias and repetitive negative thinking during pregnancy would serve as vulnerability factors for perinatal depressive symptoms and parenting stress in both mothers and fathers. We also explored gender differences.

Specifically, our hypotheses were that:

a. Repetitive negative thinking and attentional bias during pregnancy, and adverse childhood experiences, would be predictive of both parenting stress and depressive symptoms.

b. Social support, education and age would serve as protective factors for parenting stress and depressive symptoms.

As prenatal and postnatal depressive symptoms and parenting stress are related, we hypothesized that

c. Prenatal depressive symptoms would mediate effects in the relations between predictors and postnatal depressive symptoms. d. Postnatal depressive symptoms would mediate effects in the relations between predictors and parenting stress.

In a post hoc analysis between parents, with and without elevated ACE-scores, we hypothesized that

e. The elevated ACE-group would have significantly higher levels of depressive symptoms and parenting stress than the non-elevated ACE group.

\section{Method}

The present study was part of a larger study, the Northern Babies Longitudinal Study (NorBaby; Høifødt et al., 2017), that investigated pre- and postnatal risk factors for parental outcomes, parent-infant interaction and infant development.

\section{Participants}

Altogether, 220 expecting mothers and 130 of their partners were recruited as part of the NorBaby-study (Høifødt et al., 2017), totaling 350 participants. Families were followed longitudinally throughout pregnancy and after birth until the child was 6 months old. There were three measurement points during pregnancy (T1-T3) and three after birth (T4T6). In 8, data from $\mathrm{T} 1$ and $\mathrm{T} 4$ are analyzed.

\section{Analyses of Attrition}

Data from 274 participants were included in 8 . The attrition was a result of participants resigning from the study before the T4 measurement $(n=43)$, or omission of the T4 measurement $(n=33)$. The missing group was significantly different from participants in 8 when it came to gender $\left(\chi^{2}(349)=9.659, p=0.002\right.$, Cramer's V $\left.=0.166\right)$, because of a larger percentage of males in the missing group (52\%), than in the participating group (32\%). The missing group was significantly less educated $(t(346)=5.200, p<0.001$, Cohen's $d=0.681$ ), but there was no significant difference in the level of income between the groups $(t(345)=1.820$, $p=0.070, d=0.238$ ). Thus, attrition did not reflect resources and socioeconomic status. The groups did not differ in their level of depressive symptoms at $\mathrm{T} 1$, their history of depression, or amount of adverse childhood experiences (all $p$ 's $>0.300$ ).

\section{Procedure and Measures}

The first assessment (T1) in 8 was performed during pregnancy between gestational week 13 to 39 (mean week 23, median 23, SD 3.62). 93\% answered between week 18-29. The first postnatal assessment (T4) was performed 6-15 weeks after birth (mean week 8.1, median 7.7, SD 1.94). The $\mathrm{T} 1$ assessment was performed with a member 
of the research team present, whereas T4 was performed with an online survey that participants received via email 6 weeks after birth.

\section{Prenatal Assessment (T1)}

This was the first meeting between participants and a member of the research group (for details, see Høifødt et al., 2017). The relevant measures for the present study are described in detail below.

\section{Demographics}

Demographic information included gender, age, number of children, education, income, history of depression, and support from family and friends. Education was measured on an ordinal scale from low (no high school) to high (4 years or more from university/college). Income was measured ordinal for the household, from very low (below 150,000 NOK/ \$17,550) to high (above 1,000,000 NOK/117,000 USD). The average yearly income for females in Norway in 2015 (when data collection begun) was $477,600 \mathrm{NOK} / 55,878$ USD (SSB, 2016). To measure history of depression participants answered the question "Have you ever been depressed or sad almost every day, for a period of at least two weeks?". Support from family and friends were assessed with "Do you have enough friends/ family to help and support you?", with answer options yes or no.

\section{Edinburgh Postnatal Depression Scale (EPDS)}

The EPDS was developed to screen for depressive symptoms in the perinatal period (Cox et al., 1987). It consists of 10 items assessing common depressive symptoms, where each item (e.g., "In the past 7 days, I have been so unhappy that I have had difficulty sleeping") is scored from 0-3 points, with total scores ranging from $0-30$ points. Scores $\geq 10$ are commonly applied as a cutoff, indicating possible depression. In the present sample, the EPDS measured at T1 had a Cronbach's $\alpha$ of 0.81 , indicating good internal consistency.

\section{Adverse Childhood Experience Questionnaire (ACE)}

The ACE is a 10-item questionnaire that assesses whether abuse, neglect or household dysfunction were experienced during childhood. Participants answered yes or no to the ten items, and total scores range from 0-10. Felitti et al. (1998) found a strong association between ACE scores and health. They write that since the risk for a range of mental and somatic illnesses increases with each adverse childhood experience, the cumulative risk can be substantial.

\section{Perseverative Thinking Questionnaire (PTQ)}

The PTQ is a 15-item questionnaire assessing repetitive negative thinking (Ehring et al., 2011). It is a transdiagnostic questionnaire that focuses on the thought process, or how the thoughts occur, rather than the specific content of the thoughts (e.g., "The same thoughts keep going through my mind again and again"). Participants rated each item on a scale from 0 (never) to 4 (almost always), with total scores ranging from $0-60$. The scale had a Cronbach's $\alpha$ of 0.95 in the present sample, indicating high internal consistency.

\section{Emotional Dot Probe-task (EDP)}

The EDP was employed to assess attentional bias to emotional infant faces. We have described the task in previous work (Bohne et al., 2021) and the following description is similar to that. The task is based on the original dot-probe task (MacLeod, Mathews, \& Tata, 1986). Happy, sad and neutral infant faces from the Tromso Infant Faces database (Maack et al., 2017) served as stimuli. A fixation cross was presented for $500 \mathrm{~ms}$ (ms) in the middle of the screen, followed by a single stimulus image, presented for $1000 \mathrm{~ms}$. The stimulus was presented on either the left or the right side of the screen. After stimulus presentation, a dot-probe appeared on the same or the opposite side of the screen. To indicate the location of the dot-probe, participants pressed a key. The intertrial interval was $100 \mathrm{~ms}$. The stimulus (happy, sad, or neutral infant faces) and the dot-probe appeared in the left or right position with equal probability. Participants first completed 10 rehearsal trials while supervised by a member of the research group, and then 144 trials on their own. The task was programmed using Inquisit (Millisecond software), which recorded response accuracy and latency. Reliability was poor, for neutral images: $r=0.61$, for sad images: $r=0.52$ and for happy images: $r=0.59$. Note, low reliability is not seen as a hindrance to investigate differences at the group-level (Price et al., 2015; Staugaard, 2009).

\section{Postnatal Assessment (T4)}

This assessment was sent to participants by email 6 weeks after birth. The following two questionnaires were relevant for the present study.

\section{Edinburgh Postnatal Depression Scale (EPDS)}

As described above. Cronbach's $\alpha$ was 0.78 at T4, which is considered acceptable. 


\section{Parenting Stress Index - Parent Domain (PSI-PD)}

The parenting stress index (PSI; Abidin, 1983) consists of 101 items and is used to measure stress in the parent-child relation. The parent domain reflects stress in the parental role, while the child domain reflects stress associated with characteristics in the child. Together, the two domains give a total stress score. At T4, participants only answered the parent domain (54 items). Items are scored on a 5-point Likert scale, where higher scores indicate more stress. In the present sample Cronbach's $\alpha$ was 0.91 .

\section{Intervention - The Newborn Behavioral Observation (NBO)}

The intervention took place between the two measurement points in 8. After birth, 91 of the participating families were in the intervention group and received three NBO-consultations. The first consultation was at the maternity ward during the first few days after birth. The next two were provided by the well-baby clinics at a home visit 1-2 weeks after birth and at the clinic 4 weeks after birth, respectively. As Høifødt et al. (2020) found no group difference between those who received NBO and those who did not on measures included in 8 , we therefore pooled the data.

\section{Pre-Registration and Primary Data Analyses}

Analyses were planned and pre-registered on the Open Science Framework before completion of data collection (osf. io/h8379). In accordance with the pre-registered plan for analyses, we conducted regression analyses to examine significant predictors of prenatal and postnatal depression and parenting stress. When there was a violation of assumptions, we performed robust regression analysis, however the main results did not substantially change. Mediation analyses were then performed to investigate mediating effects of prenatal and postnatal depressive symptoms. Analyses were run in Jasp (JASP, 2020) and Jamovi (jamovi, 2020).

\section{Results}

\section{Sample}

A total of 274 participants ( 185 female) participated in both assessments ( $\mathrm{T} 1$ and $\mathrm{T} 4)$. The age range was from 20 to 49. Most participants had high socioeconomic status (Table 1). At $\mathrm{T} 1,9.3 \%$ experienced depressive symptoms indicating a possible depression (EPDS $\geq 10$ ). Regarding adverse childhood experiences, 15 persons (5.5\%) had 4 or more and 27 (9.9\%) had 3 or more ACE's.

The group who received the NBO-intervention differed from the group who got care as usual regarding education
Table 1 Descriptives

\begin{tabular}{lll}
\hline Variable & & $N$ \\
\hline Men / Women & $89 / 185$ & 274 \\
Age & $M=31.95(S D=4.9$, range $20-49)$ & 274 \\
Primiparous & $52.2 \%$ & 274 \\
Higher education & $87.2 \%$ & 274 \\
High income & $71.4 \%$ & 273 \\
History of depression & $28.5 \%$ & 274 \\
EPDS T1 & $M=3.82(S D=3.5$, range 0-16) & 273 \\
ACE & $M=.80$, median=0 (range 0-9) & 271 \\
\hline
\end{tabular}

Higher education $=$ university or college degree, bachelor level or higher. High income $=$ average or higher, per household $>750000$ NOK. History of depression="Have you ever been depressed or sad almost every day, for a period of at least two weeks?"

$(t(270)=2.516, p=0.012$, Cohen's $d=0.306)$, and support from family $\left(\chi^{2}(274)=5.960, p=0.015\right.$, Cramer's $\mathrm{V}=0.147)$ and friends $\left(\chi^{2}(274)=4.880, p=0.027\right.$, Cramer's $\mathrm{V}=0.134)$. The NBO group had slightly lower education and less support. The two groups did not differ from each other with respect to age, gender, parity, income, history of depression or adverse childhood experiences. Further exploration revealed that mothers in the two groups did not significantly differ on any of the demographic variables (all $p>0.050$ ).

\section{Prenatal Depression}

Five participants misunderstood the EDP-task, so the error rates were too high to include them. Another eight participants did not complete the EDP-task. Three participants did not complete the PTQ and ACE. This left 258 participants for the regression analysis.

A hierarchical bootstrapped regression model predicting prenatal depression (as measured by EPDS) was applied (Table 2). In model 1 demographic variables and ACE were entered as predictors, and in model 2 the cognitive variables (PTQ and EDP) were added. Model 1 was significant $(F(9,247)=6.378, p<0.001$; see Table 2$)$ and explained $18.9 \%$ of the variance. Five significant predictors emerged: gender $(1=$ female $)$, age, parity, family support, and history of depression. Adding the cognitive variables explained $22.4 \%$ more of the variance (model $2 ; F(12,244)=14.308$, $\Delta R^{2}=0.224, p<0.001$ ), a significant change. Significant predictors in model 2 were gender, age, education, and repetitive negative thinking, where higher age and higher education were protective against prenatal depressive symptoms.

\section{Postnatal Depression}

Three participants did not complete the PSI, leaving 255 participants for analysis. A hierarchical regression model 
Table 2 Results of hierarchical regression for predictors of prenatal depression (EPDS T1)

\begin{tabular}{llllll}
\hline Predictor variables & $\mathrm{B}$ & $\beta$ & $\mathrm{sr}^{2}$ & $\mathrm{t}$ & $\mathrm{p}$ \\
\hline Model 1 & & & & & \\
Gender & 1.613 & .223 & .207 & 3.608 & $<. \mathbf{0 0 1}$ \\
Age & -.100 & -.144 & -.116 & -2.023 & $\mathbf{. 0 4 4}$ \\
Parity & -.762 & -.155 & -.143 & -2.493 & $\mathbf{. 0 1 3}$ \\
Education & -.386 & -.082 & -.072 & -1.248 & .213 \\
Income & .105 & .043 & .038 & .671 & .503 \\
History of depression & 1.047 & .139 & .131 & 2.289 & $\mathbf{. 0 2 3}$ \\
ACE & .188 & .071 & .067 & 1.171 & .243 \\
Family support & -1.900 & -.151 & -.140 & -2.446 & $\mathbf{. 0 1 5}$ \\
Friend support & -1.091 & -.091 & -.088 & -1.532 & .127 \\
Model 2 & & & & & \\
Gender & .893 & .124 & .112 & 2.291 & $\mathbf{. 0 2 3}$ \\
Age & -.100 & -.144 & -.116 & -2.365 & $\mathbf{. 0 1 9}$ \\
Parity & -.441 & -.090 & -.082 & -1.673 & .096 \\
Education & -.548 & -.116 & -.101 & -2.064 & $\mathbf{. 0 4 0}$ \\
Income & .195 & .080 & .071 & 1.458 & .146 \\
History of depression & -.215 & -.028 & -.025 & -.518 & .605 \\
ACE & -.009 & -.004 & -.003 & -.067 & .946 \\
Family support & -.786 & -.063 & -.057 & -1.154 & .250 \\
Friend support & -.990 & -.082 & -.079 & -1.615 & .108 \\
PTQ & .192 & .551 & .470 & 9.589 & $<.001$ \\
Bias Happy & -.003 & -.033 & -.027 & -.560 & .576 \\
Bias Sad & -.003 & -.029 & -.024 & -.486 & .627 \\
\hline & & & & &
\end{tabular}

Significant $p$-values $(<.05)$ are in bold

$\mathrm{B}=$ Unstandardized beta, $\beta=$ Standardized beta, $\mathrm{sr}^{2}=$ semipartial correlation, History of depression = "Have you ever been depressed or sad almost every day, for a period of at least two weeks?", $\mathrm{ACE}=$ Adverse childhood experiences questionnaire, $\mathrm{PTQ}=$ Perseverative thinking questionnaire, bias happy/sad as measured by the EDP. Model 1: $R=.434, R^{2}=.189$, Adjusted $\mathrm{R}^{2}=.159$, Standard error $=3.093$. Model 2: $R=.643, R^{2}=.413$, Adjusted $\mathrm{R}^{2}=.384$, Standard error $=2.647$

predicting postnatal depression (as measured by EPDS) was applied (Table 3). Prenatal depressive symptoms was entered in model 1 , as it is a strong predictor of postnatal depressive symptoms. The intervention variable was also entered in model 1. In model 2, demographic and cognitive variables were entered, and finally parenting stress was entered in model 3. Results are presented in Table 3. Prenatal depressive symptoms were confirmed as a significant predictor, and model $1(F(2,251)=58.430, p<0.001)$ explained $31.8 \%$ of the variance. Model $2(F(14,239)=11.354, p<0.001)$ explained an additional $8.2 \%$ of the variance, a significant change $\left(\Delta R^{2}=0.082, p=0.002\right)$, and education and repetitive negative thinking were significant predictors in addition to prenatal depressive symptoms. Adding parenting stress improved the model significantly, increasing explained
Table 3 Results of hierarchical regression for predictors of postnatal depression (EPDS T4)

\begin{tabular}{|c|c|c|c|c|c|}
\hline Predictor variables & B & $\beta$ & $\mathrm{sr}^{2}$ & $\mathrm{t}$ & $\mathrm{p}$ \\
\hline \multicolumn{6}{|l|}{ Model 1} \\
\hline Prenatal depression & .510 & .557 & .557 & 10.676 & $<.001$ \\
\hline NBO-intervention & .512 & .082 & .082 & 1.565 & .119 \\
\hline \multicolumn{6}{|l|}{ Model 2} \\
\hline Prenatal depression & .331 & .362 & .277 & 5.526 & $<.001$ \\
\hline NBO-intervention & .414 & .066 & .063 & 1.257 & .210 \\
\hline Gender & .277 & .042 & .037 & .744 & .457 \\
\hline Age & -.014 & -.022 & -.018 & -.350 & .727 \\
\hline Parity & -.424 & -.094 & -.085 & -1.700 & .091 \\
\hline Education & -.624 & -.143 & -.122 & -2.429 & .016 \\
\hline Income & .117 & .052 & .046 & .915 & .361 \\
\hline History of depression & .527 & .076 & .068 & 1.348 & .179 \\
\hline $\mathrm{ACE}$ & .182 & .075 & .068 & 1.395 & .164 \\
\hline Family support & .065 & .006 & .005 & .100 & .920 \\
\hline Friend support & -.072 & -.007 & -.006 & -.125 & .901 \\
\hline PTQ & .071 & .222 & .161 & 3.220 & .001 \\
\hline Bias Happy & .006 & .057 & .048 & .954 & .341 \\
\hline Bias Sad & -.006 & -.064 & -.052 & -1.040 & .299 \\
\hline \multicolumn{6}{|l|}{ Model 3} \\
\hline Prenatal depression & .249 & .272 & .205 & 4.613 & $<.001$ \\
\hline NBO-intervention & .149 & .024 & .022 & .506 & .614 \\
\hline Gender & .349 & .052 & .047 & 1.056 & .292 \\
\hline Age & -.016 & -.024 & -.019 & -.436 & .663 \\
\hline Parity & -.138 & -.031 & -.027 & -.616 & .539 \\
\hline Education & -.666 & -.153 & -.130 & -2.926 & .004 \\
\hline Income & .087 & .039 & .034 & .772 & .441 \\
\hline History of depression & .227 & .033 & .029 & .654 & .514 \\
\hline $\mathrm{ACE}$ & .111 & .046 & .042 & .953 & .342 \\
\hline Family support & -.107 & -.009 & -.008 & -.186 & .852 \\
\hline Friend support & .638 & .058 & .054 & 1.223 & .223 \\
\hline PTQ & .031 & .098 & .069 & 1.557 & .121 \\
\hline Bias Happy & .003 & .028 & .024 & .532 & .595 \\
\hline Bias Sad & -.004 & -.045 & -.037 & -.825 & .410 \\
\hline Parenting stress & .065 & .439 & .363 & 8.170 & $<.001$ \\
\hline
\end{tabular}

Significant $p$-values $(<.05)$ are in bold

$\mathrm{B}=$ Unstandardized beta, $\beta=$ Standardized beta, $\mathrm{sr}^{2}=$ semipartial correlation, History of depression = "Have you ever been depressed or sad almost every day, for a period of at least two weeks?", prenatal depression $=\mathrm{EPDS}$ at $\mathrm{T} 1, \mathrm{NBO}=$ Newborn behavioral observation, $\mathrm{ACE}=$ Adverse childhood experiences questionnaire, $\mathrm{PTQ}=\mathrm{Per}-$ severative thinking questionnaire, bias happy/sad as measured by the EDP. Model 1: $R=.564, R^{2}=.318$, Adjusted $\mathrm{R}^{2}=.312$, Standard error $=2.576$. Model 2: $R=.632, R^{2}=.399$, Adjusted $\mathrm{R}^{2}=.364$, Standard error $=2.476$. Model 3: $R=.729, R^{2}=.531$, Adjusted $\mathrm{R}^{2}=.501$, Standard error $=2.193$

variance from $40 \%$ to $53.1 \%$ (model $3: F(15,238)=17.963$, $p<0.001)$. With this addition, repetitive negative thinking was no longer significant, while prenatal depressive 
symptoms and education remained significant. There was no multicollinearity (all VIFs $<2$ ).

The assumed mediational effect of prenatal depressive symptoms on the relationship between the predicted vulnerability and risk factors (repetitive negative thinking, attentional bias, adverse childhood experiences and history of depression) and postnatal depressive symptoms was investigated. First, associations between postnatal depressive symptoms and the predictors were analyzed. There was no significant association between attentional bias (as measured by the EDP-task) and postnatal depressive symptoms, and this variable was excluded from further mediation analysis. Separate bootstrapped mediation analyses for adverse childhood experiences, history of depression and repetitive negative thinking were performed. The effect of ACEs on postnatal depressive symptoms was fully mediated by prenatal depressive symptoms, while history of depression and repetitive negative thinking were only partially mediated (Fig. 1).

\section{Parenting Stress}

A hierarchical regression for predictors of parenting stress was performed (Table 4). In accordance with the chronology of assessments, demographics and cognitive factors were entered in model 1 (Model 1: $F(9$, 244) $\left.=5.709, p<0.001, R^{2}=0.174\right)$. Prenatal depressive symptoms were also measured at $\mathrm{T} 1$, but since it was established as a mediator of postnatal depressive symptoms it was entered in model 2 along with the NBO-intervention $($ Model 2: $F(14,239)=7.987$, $\left.p<0.001, R^{2}=0.319\right)$. This yielded a significant change in explained variance $\left(\Delta R^{2}=0.145, p<0.001\right)$. Postnatal depressive symptoms was added in model 3 , both because of its simultaneous assessment with the outcome and the expected suppressing effect on other variables (Model 3: $F(15,238)=13.956, p<0.001)$. Results can be seen in Table 4. The final model found support from friends being protective for parental stress whereas repetitive negative thinking and postnatal depressive symptoms had adverse effects on parental stress.

As postnatal depressive symptoms and parenting stress were highly related, we investigated the mediational effect of postnatal depressive symptoms between the assumed vulnerability factors, and parenting stress. As there was no significant association between parenting stress and attentional bias, this was left out of further mediation analysis. Three predictors were run in separate bootstrapped mediation analyses: history of depression, adverse childhood experience and repetitive negative thinking. The effect of adverse childhood experiences on parenting stress was fully mediated by postnatal depressive symptoms. The effect of history of depression and repetitive negative thinking was only partially mediated by postnatal depressive symptoms (Fig. 2).

\section{Gender Differences}

There were some important differences in demographics and cognitive factors between mothers and fathers. Mothers were significantly younger $(t(138)=3.460, p<0.001)$, and expressed higher levels of repetitive negative thinking $(t(178)=-3.400, p<0.001)$ and adverse childhood experiences $(t(245)=-2.130, p=0.034)$. Mothers were also more depressed, both according to present symptoms at $\mathrm{T} 1$ (EPDS; $t(214)=-4.710, p<0.001)$ and history of depression $\left(\chi^{2}=5.680, p=0.017\right)$.

To address these differences, we ran all regression analyses again, separate for mothers and fathers. Results for the regression analyses of predictors of prenatal depressive symptoms demonstrated that repetitive negative thinking was a highly significant predictor for both genders. For mothers, higher age, parity, and support from friends served as protective factors. For fathers, the only other significant predictor was an attentional bias for happy faces from the EDP, which was negatively related to prenatal depressive symptoms. This suggests that a tendency to disengage from happy faces is associated with higher levels of depressive symptoms in fathers.

Both prenatal depressive symptoms and parenting stress were highly significant and positively related to postnatal depressive symptoms in both mothers and fathers. History of depression was also a significant predictor in fathers, and education served as a protective factor for mothers.

Regarding predictors of parenting stress, postnatal depressive symptoms was highly significant in both mothers and fathers. Repetitive negative thinking was a significant predictor of parenting stress as well, in both genders. For mothers, these were the only significant predictors when all variables were entered. However, in fathers, support from friends was protective. See Supplementary material Tables 1-6 for full regression tables for mothers and fathers separately.

\section{Explorative Data Analyses}

To further investigate the effect of ACE on depressive symptoms, parenting stress and cognitions, we explored differences in a group comprised of participants with elevated ACE-scores $(\geq 3)$, and a matched control group. There were 27 participants scoring 3 or more on ACE. This group was matched on age, gender, education, income, number of children and support from family and friends to 27 participants (MatchIt package in R; Ho et al., 2011). $\mathrm{T}$-tests for independent samples were performed to investigate group differences. There were no differences between 
Fig. 1 Mediation models with prenatal depressive symptoms as mediator between repetitive negative thinking, adverse childhood experiences and self-reported history of depression and postnatal depressive symptoms. Coefficients are unstandardized. Total effects in brackets. $*=p<0.05$, $* *=p<0.001$
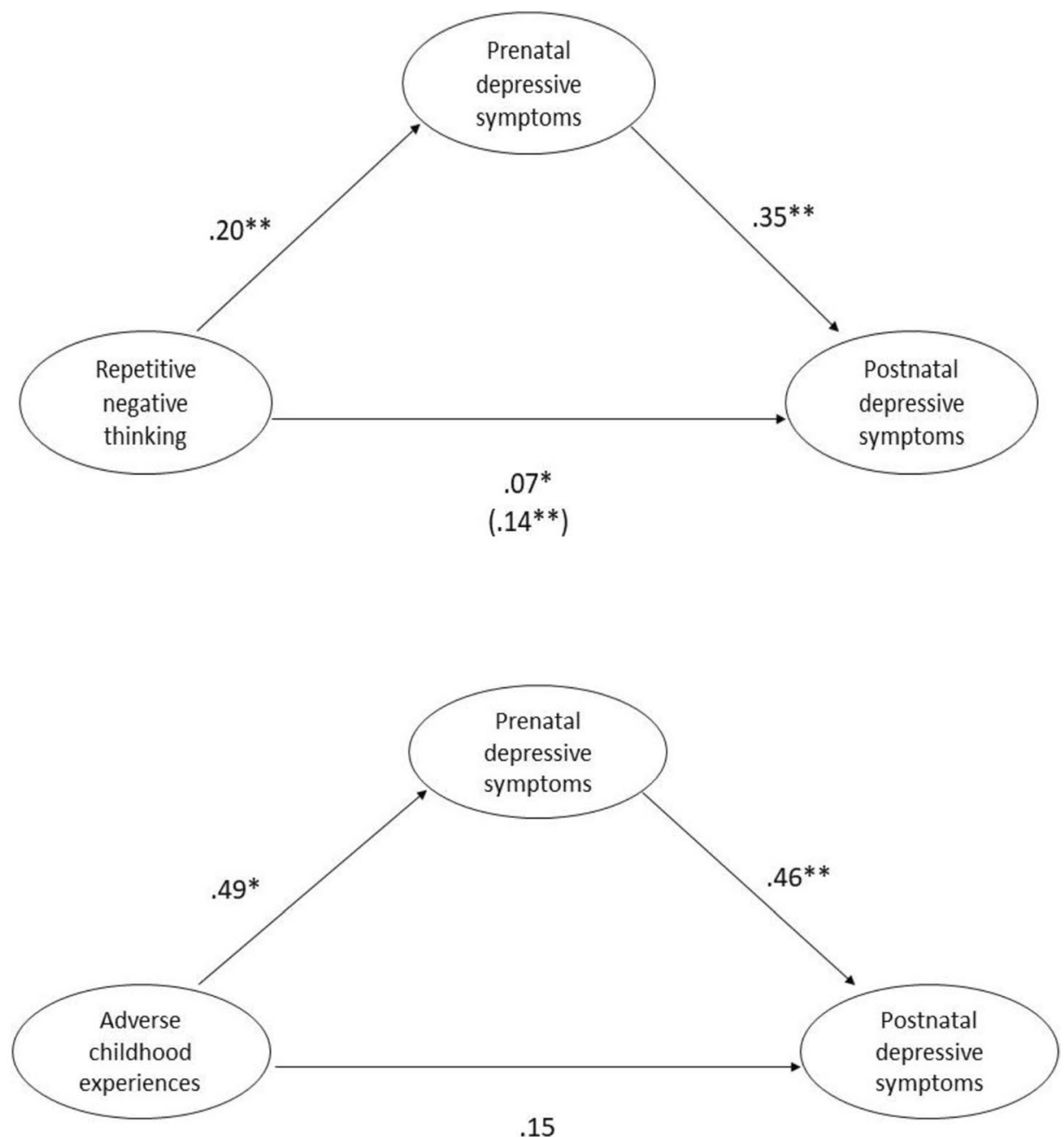

$(.37 *)$

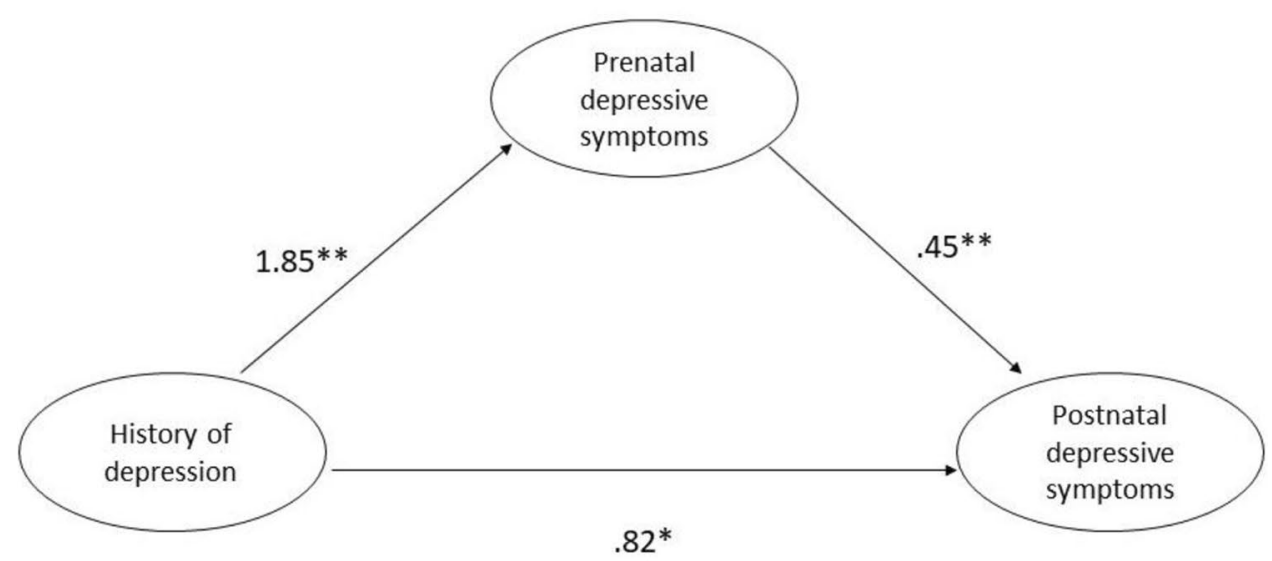

$\left(1.65^{* *}\right)$ the ACE-group and the no ACE group on either variable, all p's $>0.160$. For details, see Supplementary Material, Table 7 .

\section{Discussion}

The present study investigated predictors of parenting stress and perinatal depressive symptoms. Our results partly 
Table 4 Results of hierarchical regression for predictors of parenting stress (PSI T4)

\begin{tabular}{|c|c|c|c|c|c|}
\hline Predictor variables & B & $\beta$ & $\mathrm{sr}^{2}$ & $\mathrm{t}$ & $\mathrm{p}$ \\
\hline \multicolumn{6}{|l|}{ Model 1} \\
\hline Gender & 3.503 & .078 & .072 & 1.235 & .218 \\
\hline Age & -.089 & -.021 & -.017 & -.284 & .776 \\
\hline Parity & -.6 .215 & -.203 & -.187 & -3.219 & .001 \\
\hline Education & -.066 & -.002 & -.002 & -.034 & .973 \\
\hline Income & .463 & .030 & .027 & .466 & .642 \\
\hline History of depression & 10.224 & .217 & .206 & 3.539 & $<.001$ \\
\hline $\mathrm{ACE}$ & 1.795 & .109 & .103 & 1.773 & .077 \\
\hline Family support & -4.922 & -.063 & -.059 & -1.006 & .315 \\
\hline Friend support & -13.365 & -.179 & -.173 & -2.982 & .003 \\
\hline \multicolumn{6}{|l|}{ Model 2} \\
\hline Gender & -1.098 & -.024 & -.022 & -.408 & .683 \\
\hline Age & .023 & .005 & .004 & .077 & .938 \\
\hline Parity & -4.408 & -.144 & -.131 & -2.452 & .015 \\
\hline Education & .644 & .022 & .019 & .348 & .728 \\
\hline Income & .456 & .030 & .026 & .495 & .621 \\
\hline History of depression & 4.608 & .098 & .087 & 1.637 & .103 \\
\hline ACE & 1.104 & .067 & .063 & 1.172 & .243 \\
\hline Family support & 2.643 & .034 & .030 & .567 & .571 \\
\hline Friend support & -10.946 & -.147 & -.140 & -2.614 & .010 \\
\hline PTQ & .610 & .282 & .205 & 3.844 & $<.001$ \\
\hline Bias Happy & .043 & .066 & .055 & 1.031 & .303 \\
\hline Bias Sad & -.029 & -.043 & -.035 & -.660 & .510 \\
\hline NBO-intervention & 4.097 & .096 & .092 & 1.724 & .086 \\
\hline Prenatal depression & 1.265 & .204 & .156 & 2.925 & .004 \\
\hline \multicolumn{6}{|l|}{ Model 3} \\
\hline Gender & -2.034 & -.045 & -.040 & -.854 & .394 \\
\hline Age & .070 & .016 & .013 & .272 & .786 \\
\hline Parity & -2.978 & -.098 & -.088 & -1.859 & .064 \\
\hline Education & 2.749 & .093 & .078 & 1.656 & .099 \\
\hline Income & .061 & .004 & .004 & .075 & .940 \\
\hline History of depression & 2.831 & .060 & .053 & 1.131 & .259 \\
\hline $\mathrm{ACE}$ & .489 & .030 & .028 & .583 & .560 \\
\hline Family support & 2.424 & .031 & .028 & .587 & .558 \\
\hline Friend support & -10.701 & -.143 & -.136 & -2.885 & .004 \\
\hline PTQ & .371 & .172 & .122 & 2.584 & .010 \\
\hline Bias Happy & .025 & .037 & .031 & .659 & .511 \\
\hline Bias Sad & -.007 & -.011 & -.009 & -.195 & .846 \\
\hline NBO-intervention & 2.699 & .063 & .060 & 1.278 & .203 \\
\hline Prenatal depression & .146 & .024 & .017 & .360 & .719 \\
\hline Postnatal depression & 3.375 & .498 & .386 & 8.170 & $<.001$ \\
\hline
\end{tabular}

Significant $p$-values $(<.05)$ are in bold

$\mathrm{B}=$ Unstandardized beta, $\beta=$ Standardized beta, $\mathrm{sr}^{2}=$ semipartial correlation, prenatal depression $=$ EPDS at T1, postnatal depression $=\mathrm{EPDS}$ at $\mathrm{T} 4, \mathrm{NBO}=$ Newborn behavior observation, $\mathrm{ACE}=$ Adverse childhood experiences questionnaire, $\mathrm{PTQ}=$ Perseverative thinking questionnaire, bias happy/sad as measured by the EDP. Model 1: $R=.417, R^{2}=.174$, Adjusted $\mathrm{R}^{2}=.143$, Standard error $=19.460$. Model 2: $R=.565, R^{2}=.319$, Adjusted $\mathrm{R}^{2}=.279$, Standard error $=17.856$. Model 3: $R=.684, R^{2}=.468$, Adjusted $\mathrm{R}^{2}=.434$, Standard error $=15.813$ confirmed hypothesis a): repetitive negative thinking makes parents more vulnerable to experiencing perinatal depressive symptoms and parenting stress. However, we did not find support that attentional bias was predictive of this. Adverse childhood experiences had an indirect effect on postnatal depressive symptoms and parenting stress, fully mediated by pre- and postnatal depressive symptoms respectively. The effect of repetitive negative thinking on postnatal depressive symptoms and parenting stress was also mediated by preand postnatal depressive symptoms, although only partly, and hypothesis c) and d) were confirmed. When matched with participants without adverse childhood experiences, the group with more ACE's did equally well, thus refuting hypothesis e). Finally, results also indicated important protective factors, especially the experience of social support from friends, thus partially confirming hypothesis b).

\section{Cognitive Vulnerability}

The present and previous studies (Barnum et al., 2013; Leigh \& Milgrom, 2008) indicate that parental cognitive thinking style is important in understanding why some parents develop mental illness during the perinatal period.

It is understandable that repetitive negative thinking affects parents in the perinatal period, as DeJong et al. (2016) illustrate in their model. Minor concerns about the infant's behavior and communication, or doubt about one's own parenting, can grow into greater concerns than necessary when negative thoughts are repetitive, partly intrusive, and difficult to disengage from. Naturally, this thinking style might interfere with parents' sensitivity to their newborn child, as one of the main features of repetitive negative thinking is that it occupies mental capacity. Further, this could affect the parent-infant interaction, maybe leading to even more negative thoughts. In turn, this could predict both depressive symptoms and the experience of stress after birth. In their model, DeJong et al. (2016) also illustrate how cognitive biases, like a negative attention bias, affects what material undergoes rumination. An attentional bias can affect what you notice about your infant. Together with cognitive control deficits, such as how the thoughts are intrusive and difficult to disengage from, this pattern may be persevering. This study investigated attentional bias to baby faces. Biased attention to faces (LeMoult \& Gotlib, 2019) and baby faces (Bohne et al., 2021; Pearson et al., 2013) has been found in depressed groups, where either sad or mood-congruent faces seem to take precedence, or an emotional expression does not engage the depressed group as much as the healthy control group. This is also proposed as a possible vulnerability factor in perinatal mental illness, as in the model of DeJong et al. (2016). In 8 we did not find support for any attentional bias predicting stress or depressive symptoms in mothers. In the male group however, disengaging from happy faces 
Fig. 2 Mediation models with postnatal depressive symptoms as mediator between repetitive negative thinking, adverse childhood experiences and self-reported history of depression, and parenting stress. Coefficients are unstandardized. Total effects in brackets. $*=p<0.050, * *=p<0.001$
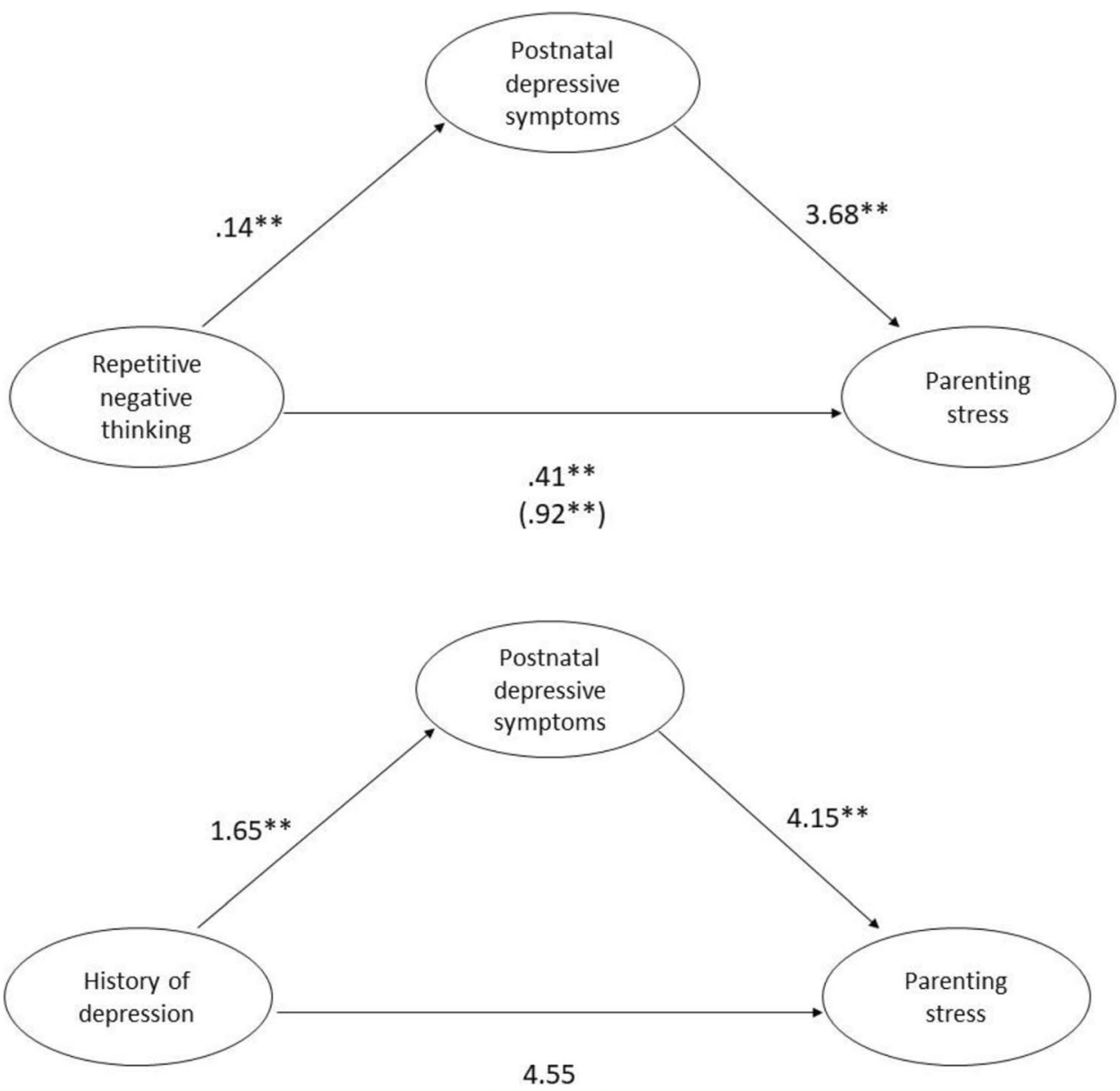

(11.41**)

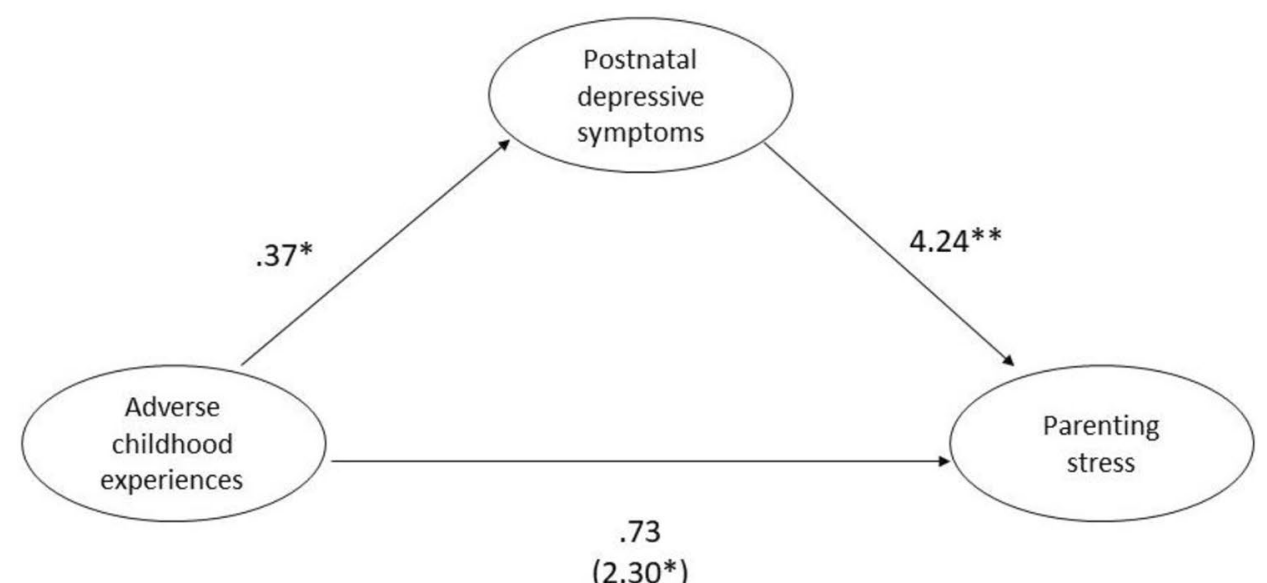

(2.30*) was related to prenatal depressive symptoms. This indicates a "protective bias", where engaging with happy infant faces might be protective of depressive symptoms.

The tendency to be caught up in negative thoughts is targeted by several treatment programs. Treatments like mindfulness-based cognitive therapy, concreteness training, cognitive control training, metacognitive therapy, rumination-focused cognitive behavioral therapy, and traditional cognitive behavioral therapy are all effective in reducing both symptoms of mental illness and repetitive negative thinking (Monteregge et al., 2020; Spinhoven et al., 2018). Offering expecting parents with high levels of repetitive 
negative thinking one of these treatment options could be preventive of further postnatal illness and parenting stress, which would also benefit the infant, and potentially break the vicious cycle.

Our results deviated from what Müller et al. (2013) found in their study, where repetitive negative thinking was predictive of mother-infant bonding, but not depressive symptoms. This inconsistency might be caused by different instruments, as Müller et al. (2013) used the Beck Depression Inventory-II (Beck et al., 1996), while we used the EPDS. The EPDS is specifically designed for depressive symptoms in the perinatal period, and therefore is less confounded by overlapping symptoms of childbirth and depression such as tiredness, lacking energy, or sexual appetite. Different instruments might be an important consideration when examining the effect of cognition on depression in the perinatal period (Fried, 2017).

\section{Protective Experience}

In line with our hypothesis d) and previous research (Norhayati et al., 2015; Racine et al., 2019, 2020), experiencing support from friends and family emerged as protective factors. While other cultures and earlier Western societies traditionally assisted and supported families in the early postnatal period, the western society today promotes an individualistic and independent lifestyle (Eckersley, 2005). This seems not to be beneficial in the perinatal period and should be noted by health care services and policy makers in their shaping of perinatal care.

The other significant protective factors in 8 have a connection with life experience: higher age, parity, and education. This might reflect how a stable life situation makes us more robust when facing challenges. Also, experience and knowledge might prepare us to meet challenging or novel situations without being overwhelmed. In their review of depression following life transitions, Moustafa et al. (2020) argue that the role change one undergoes in such a transition can cause distress if one finds it hard to accept the new role or struggles to find out what that role is. A stable life situation, often reflected in higher education and age, may make the parenting role easier to accept and adapt to in today's society. Having previous children makes the parenting role a familiar role, and one might therefore not experience a great role change.

\section{Gender Differences}

Overall, there were few differences in vulnerability between mothers and fathers. Repetitive negative thinking was a significant predictor for both genders on prenatal depressive symptoms and parenting stress. Mothers were protected by demographic factors to a higher degree than fathers, as age, parity, and social support were protective of prenatal depression, and education was protective of postnatal depression. However, this was not the case in fathers. The only demographic protective factor that was significant in fathers was support from friends on parenting stress.

\section{Resilient ACE-Group}

Contrary to what we expected, there were no group differences between those who had $\geq 3$ adverse childhood experiences and those who had none. This indicates resilience in the ACE-group and is not consistent with findings in an older ACE-study (Felitti et al., 1998). Of course, this might be caused by a participation bias, where only resilient expecting parents chose to participate. Even so, results are encouraging and underline that risk factors are not inescapable.

\section{Implications}

The current results suggest that for the sake of the parents' well-being in this transition period, prenatal care is of essence. Depressive symptoms and repetitive negative thinking during pregnancy are predictive of both postnatal parenting stress and depressive symptoms, and these postnatal conditions might affect child outcomes in a negative way (Tronick \& Reck, 2009). Both prenatal depressive symptoms and repetitive negative thinking could be addressed by effective interventions during pregnancy. To do so, early screening is needed. Identifying those with little support from friends and family and aiding in activating or building a sufficient social support network, could make a real difference for those who seem to lack it. Social support is essential for good mental health during the perinatal period (Racine et al., 2020). Health care services and policy makers need to prioritize support for expecting families, instead of downsizing. Minor, but meaningful actions in the prenatal care could make all the difference for the family facing a new life situation and the responsibility of parenting.

\section{Limitations}

As with all open population studies, the present one has an issue with participation bias. The parents that chose to participate are typically resourceful and low-risk families, with a high educational level and income, and low levels of depressive symptoms. Generalizability is limited to resourceful families. Effects might be larger in more vulnerable families, as mental illness prevalence is higher in those groups (Freeman et al., 2016). Future studies should investigate this further. 78.3\% of the participants in the study completed both T1 and T4. Attrition was high among fathers and those with lower education, which might have affected our results. 
Regarding the Emotional Dot Probe-task, our version with only a single stimulus image differs from previous research where emotional faces are shown coupled with neutral faces. This limits the comparability of the results. However, in the original version any bias towards or away from emotional images is relative to the neutral image, and therefore might have other explanations than preference or avoidance of the stimulus image. We therefore think the present version is preferable. Reliability of the task is debatable though.

Even though the EPDS is widely used as a measure of depressive symptoms in the perinatal period, it is still a short, self-report screening questionnaire, and so we cannot be certain that any of our participants experienced clinical depression. Results might differ in a clinically depressed group. This applies to our measure of previous depressive episodes as well, as this was also self-report and not based on diagnosed episodes.

\section{Conclusion}

The present study adds valuable knowledge of cognitive vulnerability factors in parents - both mothers and fathers - that is of essence in predicting perinatal depressive symptoms and parenting stress. Repetitive negative thinking is found to be a cognitive vulnerability trait in both mothers and fathers. This could be identified and targeted already during pregnancy, and thereby reduce the chance of parenting stress, worry and depressed mood in the postnatal period. Our results support vulnerability-stress models, which serve as frameworks in understanding the mechanisms involved in perinatal mental health (Ingram \& Luxton, 2005). In line with previous research, the results also highlight the importance of social support in ensuring good mental health in expecting parents. These factors seem to be of greater significance than parents' early adversity when screening for risk in the perinatal period, at least in resourceful families. The present study implicates that health and welfare services should be aware of different parental thinking styles and inquire about the social network of expecting parents. This knowledge can potentially prevent postnatal depression and parenting stress. Given the current results, interventions for expecting and new parents may be improved by enhancing social support and by adding therapeutic techniques that have been shown to reduce rumination (e.g., Spinhoven et al., 2018).

Supplementary Information The online version contains supplementary material available at https://doi.org/10.1007/s12144-021-02651-1.
Author contributions All authors were involved in the conception and design, AB, RSH, DN and IPL were involved in data acquisition, GP designed the cognitive task, $\mathrm{GP}$ and $\mathrm{AB}$ analyzed data, $\mathrm{AB}$ drafted the work and all authors critically revised and approved for publishing.

All datasets used for supporting conclusions of this article are available at the open science framework: https://osf.io/h8379/?view_only= b5317085db074679bbf38f1f7f639777

Funding Open Access funding provided by UiT The Arctic University of Norway.

\section{Declarations}

Ethics Approval The study is approved by the Regional Committees for Medical and Health Research Ethics, ref. 2015/614. We confirm that the present study complies with the 1964 Declaration of Helsinki and later addenda.

Consent to Participate Informed consent was obtained from all individual participants in the study.

Conflict of Interest On behalf of all authors, the corresponding author states that there is no conflict of interest.

Open Access This article is licensed under a Creative Commons Attribution 4.0 International License, which permits use, sharing, adaptation, distribution and reproduction in any medium or format, as long as you give appropriate credit to the original author(s) and the source, provide a link to the Creative Commons licence, and indicate if changes were made. The images or other third party material in this article are included in the article's Creative Commons licence, unless indicated otherwise in a credit line to the material. If material is not included in the article's Creative Commons licence and your intended use is not permitted by statutory regulation or exceeds the permitted use, you will need to obtain permission directly from the copyright holder. To view a copy of this licence, visit http://creativecommons.org/licenses/by/4.0/.

\section{References}

Abidin, R. R. (1992). The determinants of parenting behavior. Journal of Clinical Child Psychology, 21(4), 407-412. https://doi.org/10. 1207/s15374424jccp2104_12

Abidin, R. R. (1983). Parenting Stress Index--Manual (PSI). CPPC Tests.

Alloy, L. B., Abramson, L. Y., Smith, J. M., Gibb, B. E., \& Neeren, A. M. (2006). Role of parenting and maltreatment histories in unipolar and bipolar mood disorders: Mediation by cognitive vulnerability to depression. Clinical Child and Family Psychology Review 9https://doi.org/10.1007/s10567-006-0002-4

Barnum, S., Woody, M., \& Gibb, B. (2013). Predicting changes in depressive symptoms from pregnancy to postpartum: The role of brooding rumination and negative inferential styles. Cognitive Therapy and Research, 37(1), 71-77. https://doi.org/10.1007/ s10608-012-9456-5

Barroso, N. E., Mendez, L., Graziano, P. A., \& Bagner, D. M. (2018). Parenting stress through the lens of different clinical groups: A systematic review \& meta-analysis. Journal of Abnormal Child Psychology, 46(3), 449-461. https://doi.org/10.1007/ s10802-017-0313-6

Bauer, A., Parsonage, M., Knapp, M., Iemmi, V., Adelaja, B. (2014). Costs of perinatal mental health problems. London, UK: London School of Economics and Political Science Retrieved from https:// 
www.nwcscnsenate.nhs.uk/files/3914/7030/1256/Costs_of_perin atal_mh.pdf

Beck, A. T., Steer, R. A., \& Brown, G. K. (1996). Manual for the Beck Depression Inventory-II. (Vol. 10). Pearson.

Bergström, M. (2013). Depressive symptoms in new first-time fathers: Associations with age, sociodemographic characteristics, and antenatal psychological well-being. Birth (Berkeley, Calif.), 40(1), 32. https://doi.org/10.1111/birt.12026

Bohne, A., Nordahl, D., Lindahl, Å. A. W., Ulvenes, P., Wang, C. E. A., \& Pfuhl, G. (2021). Emotional infant face processing in women with major depression and expecting parents with depressive symptoms. Frontiers in Psychology. https://doi.org/10.3389/ fpsyg.2021.657269

Chich-Hsiu, H., Chia-Ju, L., Joel, S., \& Ching-Yun, Y. (2011). Predictors of postpartum stress. Journal of Clinical Nursing, 20(5-6), 666-674. https://doi.org/10.1111/j.1365-2702.2010.03555.x

Cox, J. L., Holden, J. M., \& Sagovsky, R. (1987). Detection of postnatal depression. Development of the 10-item Edinburgh Postnatal Depression Scale. British Journal of Psychiatry, 150, 782-786. https://doi.org/10.1192/bjp.150.6.782

DeJong, H., Fox, E., \& Stein, A. (2016). Rumination and postnatal depression: A systematic review and a cognitive model. Behaviour Research and Therapy, 82, 38-49. https://doi.org/10.1016/j.brat. 2016.05.003

Eckersley, R. (2005). Is modern Western culture a health hazard? International Journal of Epidemiology, 35(2), 252-258. https://doi. org/10.1093/ije/dyi235

Ehring, T., Zetsche, U., Weidacker, K., Wahl, K., Schönfeld, S., \& Ehlers, A. (2011). The Perseverative Thinking Questionnaire (PTQ): Validation of a content-independent measure of repetitive negative thinking. Journal of Behavior Therapy and Experimental Psychiatry, 42(2), 225-232. https://doi.org/10.1016/j.jbtep.2010. 12.003

Esteves, K. C., Jones, C. W., Wade, M., Callerame, K., Smith, A. K., Theall, K. P., \& Drury, S. S. (2020). Adverse childhood experiences: Implications for offspring telomere length and psychopathology. American Journal of Psychiatry, 177(1), 47-57. https:// doi.org/10.1176/appi.ajp.2019.18030335

Felitti, V. J., Anda, R. F., Nordenberg, D., Williamson, D. F., Spitz, A. M., Edwards, V., \& Marks, J. S. (1998). Relationship of childhood abuse and household dysfunction to many of the leading causes of death in adults: The Adverse Childhood Experiences (ACE) study. American Journal of Preventive Medicine, 14(4), 245-258. https://doi.org/10.1016/s0749-3797(98)00017-8

Fredriksen, E., von Soest, T., Smith, L., \& Moe, V. (2018). Parenting stress plays a mediating role in the prediction of early child development from both parents' perinatal depressive symptoms. Journal of Abnormal Child Psychology. https://doi.org/10.1007/ s10802-018-0428-4

Freeman, A., Tyrovolas, S., Koyanagi, A., Chatterji, S., Leonardi, M., Ayuso-Mateos, J. L., Tobiasz-Adamczyk, B., Koskinen, S., Rummel-Kluge, C., \& Haro, J. M. (2016). The role of socio-economic status in depression: Results from the COURAGE (aging survey in Europe). BMC Public Health, 16(1), 1098-1098. https://doi. org/10.1186/s12889-016-3638-0

Fried, E. I. (2017). The 52 symptoms of major depression: Lack of content overlap among seven common depression scales. Journal of Affective Disorders, 208, 191-197. https://doi.org/10.1016/j. jad.2016.10.019

Goodman, S. H., Rouse, M. H., Connell, A. M., Broth, M. R., Hall, C. M., \& Heyward, D. (2011). Maternal depression and child psychopathology: A meta-analytic review. Clinical Child and Family Psychology Review, 14(1), 1-27. https://doi.org/10.1007/ s10567-010-0080-1
Ho, D., Imai, K., King, G., \& Stuart, E. A. (2011). MatchIt: Nonparametric Preprocessing for Parametric Causal Inference. 2011, 42(8), 28. https://doi.org/10.18637/jss.v042.i08

Høifødt, R. S., Nordahl, D., Landsem, I. P., Csifcsák, G., Bohne, A., Pfuhl, G., Rognmo, K., Braarud, H. C., Goksøyr, A., Moe, V., Slinning, K., \& Wang, C. E. A. (2020). Newborn Behavioral Observation, maternal stress, depressive symptoms and the mother-infant relationship: Results from the Northern Babies Longitudinal Study (NorBaby). BMC Psychiatry, 20(1), 300. https:// doi.org/10.1186/s12888-020-02669-y

Høifødt, R. S., Nordahl, D., Pfuhl, G., Landsem, I. P., Thimm, J. C., Ilstad, L. K. K., \& Wang, C. E. A. (2017). Protocol for the Northern Babies Longitudinal Study: Predicting postpartum depression and improving parent-infant interaction with The Newborn Behavioral Observation. BMJ Open, 7(9). https://doi.org/10.1136/ bmjopen-2017-016005

Ingram, R. E., \& Luxton, D. D. (2005). Vulnerability-stress models. In B. L. Hankin \& J. R. Z. Abela (Eds.), Development of psychopathology: A vulnerability-stress perspective (pp. 32-46). Sage Publications, Inc. https://doi.org/10.4135/9781452231655.n2

jamovi. (2020). The jamovi project. In (Version 1.2)

JASP. (2020). The JASP Team. In (Version 0.12.2)

Joormann, J. (2010). Cognitive Inhibition and Emotion Regulation in Depression. Current Directions in Psychological Science, 19(3), 161-166. https://doi.org/10.1177/0963721410370293

Leigh, B., \& Milgrom, J. (2008). Risk factors for antenatal depression, postnatal depression and parenting stress. BMC Psychiatry, 8(24). https://doi.org/10.1186/1471-244X-8-24

LeMoult, J., \& Gotlib, I. H. (2019). Depression: A cognitive perspective. Clinical Psychology Review, 69, 51-66. https://doi.org/10. 1016/j.cpr.2018.06.008

Maack, J. K., Bohne, A., Nordahl, D., Livsdatter, L., Lindahl, Å. A. W., Øvervoll, M., Wang, C. E. A., \& Pfuhl, G. (2017). The Tromso Infant Faces Database (TIF): Development, validation and application to assess parenting experience on clarity and intensity ratings. Frontiers in Psychology, 8(409). https://doi.org/10.3389/ fpsyg.2017.00409

Mazzeschi, C., Pazzagli, C., Radi, G., Raspa, V., \& Buratta, L. (2015). Antecedents of maternal parenting stress: The role of attachment style, prenatal attachment, and dyadic adjustment in first-time mothers. Frontiers in Psychology, 6(1443). https://doi.org/10. 3389/fpsyg.2015.01443

Moe, V., von Soest, T., Fredriksen, E., Olafsen, K. S., \& Smith, L. (2018). The multiple determinants of maternal parenting stress 12 months after birth: The contribution of antenatal attachment style, adverse childhood experiences, and infant temperament. Frontiers in Psychology, 9(1987). https://doi.org/10.3389/fpsyg.2018.01987

Monteregge, S., Tsagkalidou, A., Cuijpers, P., \& Spinhoven, P. (2020). The effects of different types of treatment for anxiety on repetitive negative thinking: A meta-analysis. Clinical Psychology: Science and Practice, 27(2), e12316. https://doi.org/10.1111/cpsp.12316

Moustafa, A. A., Crouse, J. J., Herzallah, M. M., Salama, M., Mohamed, W., Misiak, B., Frydecka, D., Al-Dosari, N. F., Megreya, A. M., \& Mattock, K. (2020). Depression following major life transitions in women: A review and theory. Psychological Reports, 123(5), 1501-1517. https://doi.org/10.1177/00332 94119872209

Müller, D., Teismann, T., Havemann, B., Michalak, J., \& Seehagen, S. (2013). Ruminative thinking as a predictor of perceived postpartum mother-infant bonding. Cognitive Therapy and Research, 37(1), 89-96. https://doi.org/10.1007/s10608-012-9454-7

Norhayati, M. N., Nik Hazlina, N. H., Asrenee, A. R., \& Wan Emilin, W. M. A. (2015). Magnitude and risk factors for postpartum symptoms: A literature review. Journal of Affective Disorders, 175, 34-52. https://doi.org/10.1016/j.jad.2014.12.041 
Nugent, J. K., Bartlett, J. D., Von Ende, A., \& Valim, C. (2017). The Effects of the Newborn Behavioral Observations (NBO) System on Sensitivity in Mother-Infant Interactions. Infants \& Young Children, 30(4). https://journals.lww.com/iycjournal/Fulltext/ 2017/10000/The_Effects_of_the_Newborn_Behavioral_Obser vations.2.aspx

Nugent, J. K. (2007). Understanding newborn behavior \& early relationships : the newborn behavioral observations (NBO) system handbook. Paul H. Brookes.

Östberg, M., \& Hagekull, B. (2000). A structural modeling approach to the understanding of parenting stress. Journal of Clinical Child Psychology, 29(4), 615-625. https://doi.org/10.1207/S15374424J CCP2904_13

Pearson, R. M., Cooper, R. M., Penton-Voak, I. S., Lightman, S. L., \& Evans, J. (2010). Depressive symptoms in early pregnancy disrupt attentional processing of infant emotion. Psychological Medicine, 40(04), 621-631. https://doi.org/10.1017/S0033291709990961

Pearson, R. M., Lightman, S. L., \& Evans, J. (2011). Attentional processing of infant emotion during late pregnancy and mother-infant relations after birth. Archives of Women's Mental Health, 14(1), 23-31. https://doi.org/10.1007/s00737-010-0180-4

Pearson, R. M., O’Mahen, H. A., Burns, A., Bennert, K., Shepherd, C., Baxter, H., Chauhan, D., \& Evans, J. (2013). The normalisation of disrupted attentional processing of infant distress in depressed pregnant women following Cognitive Behavioural Therapy. Journal of Affective Disorders, 145(2), 208-213. https://doi.org/10. 1016/j.jad.2012.07.033

Price, R. B., Kuckertz, J. M., Siegle, G. J., Ladouceur, C. D., Silk, J. S., Ryan, N. D., Dahl, R. E., \& Amir, N. (2015). Empirical recommendations for improving the stability of the dot-probe task in clinical research. Psychological Assessment, 27(2), 365. https:// cloudfront.escholarship.org/dist/prd/content/qt2fr2s588/qt2fr 2s588.pdf?t=ozl381

Racine, N., Plamondon, A., Hentges, R., Tough, S., \& Madigan, S. (2019). Dynamic and bidirectional associations between maternal stress, anxiety, and social support: The critical role of partner and family support. Journal of Affective Disorders, 252, 19-24. https://doi.org/10.1016/j.jad.2019.03.083

Racine, N., Zumwalt, K., McDonald, S., Tough, S., \& Madigan, S. (2020). Perinatal depression: The role of maternal adverse childhood experiences and social support. Journal of Affective Disorders, 263, 576-581. https://doi.org/10.1016/j.jad.2019.11.030

Ramchandani, P., Stein, A., Evans, J., Amp, A., \& Connor, T. G. (2005). Paternal depression in the postnatal period and child development: A prospective population study. The Lancet,
365(9478), 2201-2205. https://doi.org/10.1016/S0140-6736(05) 66778-5

Sanders, L. W., \& Buckner, E. B. (2006). The Newborn Behavioral Observations system as a nursing intervention to enhance engagement in first-time mothers: Feasibility and desirability. Pediatric Nursing, 32(5), 455-459.

Schmidt, D., Seehagen, S., Hirschfeld, G., Vocks, S., Schneider, S., \& Teismann, T. (2017). Repetitive Negative Thinking and Impaired Mother-Infant Bonding: A Longitudinal Study. Cognitive Therapy and Research, 41(3), 498-507. https://doi.org/10.1007/ s10608-016-9823-8

Skjothaug, T., Smith, L., Wentzel-Larsen, T., \& Moe, V. (2014). Prospective fathers' adverse childhood experiences, pregnancyrelated anxiety, and depression during pregnancy. Infant Mental Health Journal, 36(1), 104-113. https://doi.org/10.1002/imhj. 21485

Skjothaug, T., Smith, L., Wentzel-Larsen, T., \& Moe, V. (2018). Does fathers' prenatal mental health bear a relationship to parenting stress at 6 months? Infant Mental Health Journal, 39(5), 537-551. https://doi.org/10.1002/imhj.21739

Spinhoven, P., Klein, N., Kennis, M., Cramer, A. O. J., Siegle, G., Cuijpers, P., Ormel, J., Hollon, S. D., \& Bockting, C. L. (2018). The effects of cognitive-behavior therapy for depression on repetitive negative thinking: A meta-analysis. Behaviour Research and Therapy, 106, 71-85. https://doi.org/10.1016/j.brat.2018.04.002

SSB. (2016). Income, 2015. Statistics Norway. Retrieved 20.10.2020 from

Staugaard, S. R. (2009). Reliability of two versions of the dot-probe task using photographic faces. Psychology Science, 51(3), 339350. https://psycnet.apa.org/record/2009-21519-006

Thompson-Booth, C., Viding, E., Mayes, L. C., Rutherford, H. J. V., Hodsoll, S., \& McCrory, E. J. (2014). Here's looking at you, kid: Attention to infant emotional faces in mothers and non-mothers. Developmental Science, 17(1), 35-46. https://doi.org/10.1111/ desc. 12090

Tronick, E., \& Reck, C. (2009). Infants of depressed mothers. Harvard Review of Psychiatry, 17(2), 147-156. https://doi.org/10.1080/ 10673220902899714

Publisher's Note Springer Nature remains neutral with regard to jurisdictional claims in published maps and institutional affiliations. 